oesophageal stricture is present causing dysphagia. The patient should avoid large meals, constricting clothing, and-so far as possible-bending and stooping; and he should keep his head and shoulders propped up in bed. Of the wide range of drugs claimed to relieve symptoms, either the long-acting antacid aluminium hydroxide or the muscle relaxant metoclopramide should be tried. For the overweight patient, losing weight alone may greatly diminish or even abolish virtually all his symptoms.

Surgery, then, is indicated only for the minority whose symptoms cannot be controlled by these measures, or who have a fibrous stricture. Formerly the main object of operation was to achieve as precise an anatomical repair of the hiatus and the oesophagogastric junction as possible. Many tricks were devised to bring the stretched edges of the right crus of the diaphragm together by stitches either in front of or behind the oesophagus. Surgical ingenuity also devised means of restoring the oesophagogastric angle and fixing this to the under surface of the diaphragm, the posterior abdominal wall, or other intra-abdominal organs. Various ligaments were repaired, fascial or muscular slings were created, and a variety of valvular structures were arranged.

Such repairs were not, however, always permanent or indeed successful in relieving symptoms. It now appears that restoring the correct pressure patterns is much more important than exact anatomical repair of the hiatus. Many fine studies of the pattern of pressure waves in the oesophagus have shown that a segment at the lower end has an increased pressure, and acts normally as an efficient barrier against any appreciable reflux from the stomach. But this barrier works efficiently only if hydrostatic pressure is greater in the abdominal cavity than in the chest. The main object of surgical procedures for sliding hiatus hernia therefore should be to secure a continuous subdiaphragmatic location for this lower oesophageal sphincter zone-possibly the major common factor underlying the success of the variety of operations introduced in the last 20 years or so.

One of the simplest ways of achieving this object is plicating the fundus. Nissen's method, ${ }^{2}$ for example, is a relatively simple manoeuvre creating a "bung" to discourage further dislocation upwards of the lower oesophagus through the lax hiatus. Further refinements may or may not be necessary. ${ }^{3} \mathrm{~A}$ similar procedure is the posterior gastropexy described by Hill. ${ }^{+}$Where an appreciable stricture, oesophagitis, or perioesophagitis is present the oesophagus may have to be so far mobilised that a transthoracic approach is needed." "Most uncomplicated oesophageal reflux defects, however, can probably be repaired adequately by one of the fundoplication procedures. Nissen's fundoplication may be satisfactory in over $80^{\prime \prime}$ " of patients with some stricture, and in $85-90^{\prime \prime}$ " of patients with no stricture.

The operation occasionally produces dysphagia owing to over-zealous tightening of the fundus wrap round the lower end of the oesophagus. This can be largely avoided, however, by putting a large-bore nasogastric tube in the lumen of the lower oesophagus during operation; when it does occur it is usually relieved by one or more gentle dilatations through the oesophagoscope. Patients may also have difficulty in belching or in vomiting after this operation, and the formation of a muscular sling or lesser or greater curve valve has therefore been advocated. Whether these procedures have any appreciable advantage over the simpler form of fundoplication remains to be seen.

Surgeons have always been reluctant to excise oesophageal strictures, as even the most skilful and ingenious of reconstruc- tions have been followed by a high incidence of further strictures, ulcerations, and other problems: perhaps the subsequent regurgitation of bile has caused as much harm as acid gastric juice. The alternative approach of preoperative bougie dilatation of the stricture followed by one of the forms of antireflux fundoplication appears to work in most cases.

Experimentally, gastrin and other hormones of the upper gastrointestinal tract have a pharmacological effect on the lower oesophageal musculature. There is, however, no evidence that they have any important role in sliding hiatus hernia, and thus no justification for any operations on the stomach designed to influence their secretion. Neither is there good evidence that any form of vagotomy with or without gastric drainage procedures benefits sliding hiatus hernia. Indeed, controlled trials of vagotomy as an accompaniment to the direct surgical treatment of the hiatus hernia have shown a greater incidence of postoperative problems in the vagotomy group. ${ }^{8}$

The surgical treatment of sliding hiatus hernia now appears to be on a sounder basis. We have learned to resort to surgery only when there are clear symptoms of oesophageal reflux, and after conscientiously applied medical measures have failed to bring relief. We now know that restoring the lower oesophageal high-pressure zone to a position below the diaphragm is far more important than precise anatomical repair of the hiatus, a sometimes futile objective. The vogue for plicating the fundus through an abdominal approach is likely to continue, though long, severe strictures and perioesophageal inflammation will still call for a transthoracic approach. Controlled trials would show whether the many variants of straightforward fundoplication, using slings, valves, and various methods of fixation to surrounding structures, have any real advantage over the simpler operations. There may still be scope for improving the already high success rates of surgery for sliding hiatus hernia.

\footnotetext{
${ }^{1}$ Bingham, J A W, British fournal of Surgery, 1977, 64, 460.

${ }^{2}$ Nissen, R, American Fournal of Digestive Diseases, 1961, 6, 954.

Sifers, E C, et al, Surgery, Gynecology, and Obstetrics, 1976, 143, 376.

${ }^{4}$ Hill, L D, Annals of Surgery, 1967, 166, 681.

"Collis, J L, Thoraxchirurgie, 1963, 11, 57.

'Baue, A E, and Belsey, R H R, Surgery, 1967, 62, 396.

Franklin, R H, Iweze, F I, and Owen-Smith, M S, British Fournal of Surgery, 1973, 60, 65 .

Vansant, J H, and Baker, J W, Annals of Surgery, 1976, 183, 629.
}

\section{A new approach to thrombosis?}

The ubiquitous prostaglandins tended to be hailed on their discovery as the basis for revolutionary drugs, and the prostaglandins active in blood vessels were no exception. But here, as in all the hest stories, there turn out to be "good" and "bad" prostaglandins, confusing an already complicated plot.

We do not know why thrombi develop in human arteries and veins, nor do we know by what mechanisms they are formed. We do know that thrombi contain masses of blood platelets surrounded by fibrin and polymorphonuclear leucocytes, ${ }^{1}$ and that the earliest event observed when small vessels are damaged in animals is the adherence of blood platelets to the site of injury." One view of thrombus formation is that once the protective overiying endothelial cells have been damaged or removed a sequence of events is initiated because the subendothelial tissues are thereby exposed to circulating platelets. This theory proposes that platelets adhere to the site and then release chemical messengers that will cause more platelets to 
adhere to them, the growing thrombotic mass thus being formed by a chemically mediated chain reaction or amplifying system. ${ }^{3}$

This process cannot be studied in man, but in injured animal arteries a complex series of interlinked chemical reactions clearly does occur. When platelets are challenged by thrombin or collagen in vitro they not only release the powerful aggregating agent adenosine diphosphate (ADP) but also make available prostaglandin $\mathrm{E}_{2}$, the prostaglandin endoperoxides $\mathrm{PGG}_{2}$ and $\mathrm{PGH}_{2}$, and the thromboxane $\mathrm{A}_{2}$ (the most active platelet-aggregating substance so far identified). ${ }^{4}$ These aggregatory materials are produced from platelet arachidonic acid under the influence of the enzyme cyclo-oxygenase. It has been suggested that this potentially thrombotic system is kept under control in healthy blood vessels by an equally powerful antiaggregant prostaglandin, known as $\mathrm{PGI}_{2}$ (prostacyclin or prostaglandin $\mathrm{X}$ ), produced by intact endothelium. ${ }^{5}$

Platelet activity, both in vitro and in animal models of thrombus formation, ${ }^{6}{ }^{7}$ is also powerfully inhibited by the prostaglandin $\mathrm{PGE}_{1}$. Could this then be used as an antithrombotic agent ? Unfortunately, like all the prostaglandins, it is rapidly broken down in the body, and during its brief sojourn in the circulation it produces not only effects on platelet behaviour but also side effects. ${ }^{8}$ Platelets produce $\mathrm{PGE}_{1}$ from di-homo- $\gamma$-linolenic acid (DHLA); since therefore the "bad" prostaglandins derive from arachidonic acid (AA) and the "good" ones from DHLA, conceivably we could make the platelets less reactive, and therefore less likely to initiate or take part in thrombus formation, simply by increasing the platelet DHLA:AA ratio.

Testing this hypothesis is unlikely to be quick or easy, but the paper by McNicol and his colleagues (p 1441) reports some initial observations on the feasibility of the manoeuvre. They showed that adding DHLA to platelets in vitro caused appreciable alterations in behaviour. When DHLA was given to human volunteers, their platelets did indeed contain more of the "good" DHLA than the "bad" AA, and they synthesised more of the "good" prostaglandin $\mathrm{E}_{1}$. Disappointingly, however, they also synthesised more of the "bad" prostaglandin $\mathrm{PGE}_{2}$. Moreover, in vitro tests on platelets from those who had received DHLA did not show any very striking or consistent decrease of aggregation. The heparin-neutralising activity of their plasma, however, was considerably reduced. This plasma activity is thought to be related to platelet Factor 4, but its role in causing or detecting thrombosis has not been established.

The extent to which changes in the DHLA:AA or "good":"bad" prostaglandin ratio will prove antithrombotic depends on what part these products of platelets actually play in thrombosis. Although a thrombus contains adherent and aggregated platelets, we cannot assume that mechanisms identified in vitro necessarily take part in thrombus formation. Adhesion, aggregation, and the release reaction can be studied only in shed blood to which anticoagulants have been added; so we do not yet know the precise relationship between these phenomena and platelet activities in the body, whether normal or abnormal. Trials of agents and regimens that modify the platelet prostaglandin mechanisms must be carried out before we can tell whether the results obtained by $\mathrm{McNicol}$ and his colleagues have any clinical application. There is still much to discover about the aetiological and pathogenetic mechanisms of thrombosis; but, as with insulin and diabetes, we may evolve ways of correcting an abnormality long before we understand the complexities that underlie it.
${ }^{1}$ Poole, J C F, and French, J E, Fournal of Atherosclerosis Research, 1961, $1,251$.

2 Bizzozero, J, Virchows Archiv für Pathologische Anatomie und Physiologie und für Klinische Medizin, 1882, 90, 261.

${ }^{3}$ Baumgartner, H R, Thrombosis et Diathesis Haemorrhagica, 1974, 59, (supplement), 91.

${ }^{4}$ Hamberg, M, Svensson, J, and Samuelsson, B, Proceedings of the National Academy of Sciences of the United States of America, 1975, 72, 2994.

${ }^{5}$ Moncada, S, et al, Nature, 1976, 263, 663.

${ }^{6}$ Kloeze, J, in Prostaglandins; Proceedings of Second Nobel Symposium, ed $\mathrm{S}$ Bergström and B Samuelsson. Stockholm, Almqvist and Wiksell, 1967.

Emmons, P R, et al, British Medical fournal, 1967, 2, 468.

" Elkeles, R, et al, Lancet, 1969, 2, 111.

\section{Dominant transmission of mental handicap}

We still have no idea what causes severe mental handicap in a high proportion of cases. Thus in one large hospital series it was ascribed to genetic factors in only about one-quarter of cases. ${ }^{1}$ Another community-based study ${ }^{2}$ attributed half the cases to genetic causes, but in both these series visible chromosome errors predominated. Only a small fraction of heritable forms of mental handicap are due to mutant genes. Of these most are, like phenylketonuria, autosomal recessive conditions; some are identified sex-linked conditions such as Hunter's syndrome; and others are grouped as syndromes of familial subnormality. ${ }^{34}$ While there are many dominantly transmissible conditions which may affect intelligence, all are uncommon: together they account for only a very small fraction of the total of mentally handicapped individuals.

At first sight genetic counselling might seem particularly important for families with someone affected with mental handicap of this kind in view of the high theoretical risk with a Mendelian dominant trait (and, indeed, all families with a mentally handicapped member should have access to genetic counselling). In practice, however, the risk to the rest of the family of a patient with a condition such as tuberous sclerosis is much less than might be thought, since most cases are due to a new mutation-61 out of 71 cases in one series. ${ }^{5}$ All of the 10 affected parents who had transmitted this disease had the classical features of it, including adenoma sebaceum. In one case outside that series a parent without adenoma sebaceum but with a shagreen patch transmitted tuberous sclerosis to his three children. ${ }^{6}$ The minority of mildly affected individuals with this condition who are fertile run a $50 \%$ risk of transmitting the disease, probably in a severe form, to their offspring. The gloomy genetic prognosis for this group of patients is compounded because at present we have no biochemical means of detecting the disease, and hence prenatal diagnosis is impossible. Careful examination of parents of an affected child is therefore essential, and this should include a search for adenoma sebaceum and scrutiny of the whole body for a shagreen patch; some weight might be attached to "white naevi."

Some other dominantly transmitted conditions (such as the central form of neurofibromatosis) are only occasionally complicated by mental handicap. In these circumstances genetic counselling is not so much concerned with the risk of mental handicap as with complications such as neuromata and general cosmetic considerations.

In acrocephalosyndactyly (as in tuberous sclerosis) the severity of the abnormality usually precludes transmission, but in this and Marfan's syndrome the condition is known to be 